\title{
Germanica
}

\section{Casanova : l'impossible retour à Venise}

dans L'Aventurier et la Cantatrice de Hofmannsthal et Le retour de Casanova de Schnitzler

Casanova - Venedig oder die unmoögliche Heimkehr in Der Abenteurer und die Sängerin von Hugo von Hofmannsthal und Casanovas Heimfahrt von Arthur Schnitzler

\section{Geneviève Roussel}

\section{(2) OpenEdition}

\section{Journals}

Édition électronique

URL : http://journals.openedition.org/germanica/2802

DOI : 10.4000/germanica.2802

ISSN : 2107-0784

Éditeur

Université de Lille

\section{Édition imprimée}

Date de publication : 30 juin 1987

Pagination : 7-34

ISSN : 0984-2632

\section{Référence électronique}

Geneviève Roussel, «Casanova : l'impossible retour à Venise », Germanica [En ligne], 1 | 1987, mis en ligne le 30 mars 1987, consulté le 06 octobre 2020. URL : http://journals.openedition.org/germanica/ 2802 ; DOI : https://doi.org/10.4000/germanica.2802

Ce document a été généré automatiquement le 6 octobre 2020.

(c) Tous droits réservés 


\title{
Casanova : l'impossible retour à Venise
}

\author{
dans L'Aventurier et la Cantatrice de Hofmannsthal et Le retour de \\ Casanova de Schnitzler \\ Casanova - Venedig oder die unmoögliche Heimkehr in Der Abenteurer und \\ die Sängerin von Hugo von Hofmannsthal und Casanovas Heimfahrt von \\ Arthur Schnitzler
}

Geneviève Roussel

1 Faire rentrer Casanova à Venise: sujet tentant pour un écrivain qu'attirent le personnage de Casanova et la Venise du dix-huitième siècle. Casanova lui-même étant resté fort discret sur ce sujet, l'écrivain a toute liberté pour narrer cet épisode singulier dans la vie du séducteur. Hugo von Hofmannsthal et Arthur Schnitzler, qui, à plusieurs reprises, ont choisi Casanova pour personnage principal de leurs œuvres, ont tous deux, à une vingtaine d'années d'intervalle, raconté à leur façon ce retour.

Le jeune Hugo von Hofmannsthal imagine, à partir d'un épisode des Mémoires, le retour incognito à Venise d'un Casanova de quarante ans, dans sa pièce de 1899, L'Aventurier et la Cantatrice ${ }^{1}$. Arthur Schnitzler, vingt ans plus tard, alors qu'il a lui-même cinquante ans, fait rentrer, dans sa longue nouvelle Le retour de Casanova ${ }^{2}$ un Casanova de cinquante-trois ans, qui a décidé de finir ses jours dans sa patrie.

Hofmannthal et Schnitzler qui, ailleurs, ont mis en scène Casanova séducteur, ou émanation d'une grande époque de Venise, s'attachent ici à Casanova Aventurier. Dans la pièce de Hofmannsthal tous les autres acteurs portent le nom de personnages des Mémoires et sont en grande partie conformes à ces personnages ; Casanova, qui n'est jamais nommé, est donc bien le héros de la pièce, mais en tant qu'Aventurier, mythe universel. Schnitzler, lui, insiste, dans l'introduction et la conclusion qui enferment sa nouvelle, sur ce personnage de l'Aventurier vieillissant, puis vieux.

4 L'aventurier qui rentre dans la patrie de sa jeunesse n'est pas un voyageur banal. Pour Casanova l'exil a été une contrainte - il a fui Venise où il est condamné pour crime de 
libre pensée -, mais génératrice de nouvelles aventures et de nouveaux exploits. Son retour est de nature particulière, car nulle Pénélope, nul royaume ne l'attendent.

Ce retour sera un rendez-vous de l'aventurier avec lui-même. Un huis clos de quarantehuit heures aura pour dénouement, plus léger chez Hofmannsthal, plus dramatique chez Schnitzler, la disparition de l'aventurier. Le retour est impossible pour le héros de quarante ans, que la servitude de l'aventure entraînera ailleurs, loin de son "double » idéal, son fils retrouvé à Venise, qui y perpétuera le mythe de Casanova. Le vieil homme qui, au terme de la nouvelle de Schnitzler, s'endort enfin à Venise après vingt-cinq ans d'exil, n'est plus que la dépouille de Casanova, mort symboliquement par deux fois. En se reniant lui-même pour pouvoir rentrer dans sa ville natale ; en tuant son double et rival en duel - le Casanova de la jeune génération - : castration du ms, qui entraîne la mort du mythe.

\section{L'Aventurier aux mille patries Hugo von Hofmannsthal}

6 "Un aventurier, sous le nom de baron Weidenstamm », rentre, après plus de quinze ans d'absence, "vers le milieu du dixhuitième siècle, à Venise " ${ }^{3}$, où il retrouve par hasard Vittoria, une cantatrice aimée et abandonnée autrefois, et leur fils Cesarino, dont il ignorait la naissance - telle est l'intrigue de la pièce de Hofmannsthal. Il se fait passer pour un gentilhomme d'Amsterdam - la Venise du Nord! -, riche, nanti de lettres de crédit de banquiers viennois, ayant beaucoup voyagé en Europe, séjourné dans les villes et cours les plus diverses, qui vient s'installer à Venise, où il semble décidé à mener une vie conforme à son rang. Il a loué un palais, chargé son valet Le Duc de recruter du personnel. Il passe ses soirées à l'Opéra, côtoie la noblesse, réunit dans son salon autour d'une table de jeux les représentants disparates de la société vénitienne. Il noue simultanément plusieurs intrigues amoureuses, et avec tous se montre généreux, voire fastueux.

7 Le baron, qui parle un vénitien parfait et connaît admirablement la ville, est en fait Casanova, aimé par Vittoria sous le prénom d'Antonio, qui, quinze ans plus tôt, quittait Venise en s'évadant des Plombs, matelassé de vêtements variés superposés pour amortir sa chute. Hofmannsthal, lecteur assidu des Mémoires, reprend ce trait, ou bien tel autre, non par souci de vérité historique, mais parce qu'ils apportent vie au type mythique de l'Aventurier ${ }^{4}$, tout en prenant valeur de symboles.

8 La première caractéristique de l'aventurier est l'énergie, l'appétit de vivre. Ce n'est pas le pouls de la vie qu'il entend battre en lui, mais «l'indomptable feu de son sang». L'univers est quelque chose sur quoi « il lui faut se jeter de toute la force de ses sens", dont il veut, " comme une éponge, s'imprégner $»^{5}$. L'aventurier saisit la vie à pleines mains, pour le plaisir - le mot Lust revient à tout instant dans sa bouche -; il est tout différent de ceux qui regardent la vie, les frileux, telle jeune Salaino, qui n'ose rien en amour, ne risque rien en affaires, ou Venier, le sénateur, époux de Vittoria, qui examine chacun à travers son lorgnon et souffre de spleen. Différent aussi des artistes qui certes subliment la vie, mais travaillent un «matériau mort", tel le sculpteur le marbre, Vittoria son chant $^{6}$. Cette boulimie ne va pas sans quelque vanité, quelque emphase. Sa langue foisonne d'images, de références, de superlatifs et d'hyperboles. Ne prétend-il pas goûter la vie avec « sept sens »! Il charge son valet de « recruter non pas 
trois, pas cinq, mais dix serviteurs ", il veut organiser pour ses retrouvailles avec Venise des fêtes plus luxueuses que celles du doge et de la mer.

9 La vitalité de l'aventurier se manifeste dans son goût du jeu, du masque, du risque. Le monde est un théâtre où les personnages se succèdent comme des "marionnettes ", lui-même étant l'Arlequin de la farce ou un "roi de jeu de cartes", qui aime bouleverser le destin d'autrui ${ }^{7}$ et voir " danser le hasard, ce dieu exubérant (qui) sème le désordre ». Vivre c'est jouer, gagner - l'argent, l'amour permettent de chatouiller le destin-, "parer les coups", risquer; le retour n'est pas le moindre de ces risques, Venise la tentatrice pouvant se révéler, il le sait, un fatal " piège à rats ».

Une des formes les plus subtiles du jeu est le jeu de la séduction. Alors que Don Juan collectionne les preuves de son pouvoir, l'aventurier charme naturellement tout un chacun homme, femme, enfant, vieillard, marchand, patricien ou courtisane -, par son art du compliment, son éloquence, et surtout parce qu'il apprécie d'emblée son interlocuteur à sa juste valeur et sait, à parts égales, et recevoir et donner8.

11 L'aventurier « saisit aux cheveux la volage déesse Occasion », toujours unique, toujours renouvelée. S'il se pose sur un des " rayons de la roue du destin », c'est « le temps d'un souffle », car « la hâte est la plus belle des ivresses », " attendre, c'est l'enfer »'. Chaque instant est totalité : l'épuiser, c'est s'accomplir et prendre un nouvel élan ${ }^{10}$.

12 Voyageur sans patrie, messager du destin, allié du vent, volage et voleur, Weidenstamm-Casanova vole de par le monde, « des ailes aux pieds », Hermès d'opérabouffe ou des Mille et Une Nuits ${ }^{11}$.

\section{L'Amant de Venise Hugo von Hofmannsthal}

L'itinéraire d'un aventurier n'est pas voyage de formation, initiation, pas plus qu'ascension ou Odyssée : les étapes en sont régies par le hasard et le plaisir. Pourquoi donc, vers quarante ans, Casanova rentre-t-il à Venise, où rien ni personne ne l'attend ; où il court le danger d'être reconnu et livré à l'Inquisition? Quand ce danger se fait menaçant, il s'interrogera, mais sans pouvoir répondre ${ }^{12}$.

14 L'étape de Venise serait-elle donc une aventure à laquelle il ne saurait, pas plus qu'aux autres, fixer d'avance durée ou terme ? L'organisation du train de vie indiquerait à tout le moins un séjour prolongé, mais en même temps, comme pour écarter toute mainmise sur son avenir, il déclare avoir «l'intention de séjourner ici quelques jours seulement $»$.

15 S'agirait-il, inconsciemment, de faire le point avant de nouveaux départs ? De constater que l'on n'a pas été oublié13 ? Mais la douceur du revoir, Weidenstamm ne peut y prétendre, puisque "personne ici ne (le) connaît", qu'il voyage sous une fausse identité. Plaisir de vérifier une certaine réussite sociale à l'endroit même que l'on a quitté misérable? Ou de retrouver un lieu lié à des temps forts de la vie, la fameuse fuite des Plombs, les heures de bonheur avec Vittoria ${ }^{14}$ ?

16 Aucun de ces mobiles ne ramène Casanova à Venise, pas plus que la nostalgie de la patrie, de la terre natale, le désir de retrouver ses racines. L'étranger, l'inconnu sont le sol nourricier du vagabond. L'Europe est devenue sa maison, le monde son jardin, et c'est le désir qui lui crée des patries ${ }^{15}$. 
17 L'aventurier aux mille patries rentre à Venise par amour. Non pas l'amour d'un fils pour la mère-patrie, d'un poète pour Venise la Belle, mais une violente pulsion de désir pour la bien-aimée. À son entrée en scène, il s'écrie : «j'aime cette ville par dessus tout ! $»^{16}$.

18 Les Vénitiens sont vénitiens par leur identité sociale, leur pouvoir sur la ville, leur rôle traditionnel. Lui est à la fois pécheur et doge, sénateur et mendiant, danseur et prisonnier, "dix fois plus vénitien » que chacun d'eux, parce qu'il goûte cette ville comme une maîtresse dont on sait la saveur ${ }^{17}$. Vantant sa bien-aimée, idéalisant sa féerie, il évoque sa naissance mythique lors d'une nuit fabuleuse, s'enivre de ses charmes de déesse alanguie ${ }^{18}$, rêve de célébrer leurs retrouvailles en une fête plus somptueuse encore que les épousailles du doge et de la $\operatorname{mer}^{19}$. Quand on lui fait remarquer qu'il a pour Venise l'éloquence d'un poète, il rectifie : «celle d'un amant, tout au plus d'un amant $»^{20}$.

19 Faisant le bilan de sa première journée, le baron recense les désillusions du retour et constate que Venise n'en fait pas partie ${ }^{21}$. À la déclaration d'amour liminaire répond en écho le cri de l'amant comblé !

\section{«Tel un oiseau qui pour mourir »... Arthur Schnitzler}

Le Casanova de Schnitzler n'a guère d'autre point commun avec celui de Hofmannsthal que le passé. L'humeur vagabonde qui « le chasse à travers le monde » n'est plus « goût de l'aventure propre à la jeunesse ", mais "instabilité de la vieillesse qui approche " ${ }^{22}$. Aventurier de cinquante-trois ans, à l'éclat finissant, aux moyens financiers étriqués, il voyage sans équipage, se loge dans de modestes auberges, porte du linge au luxe rapiécé et deux costumes fatigués, seuls restes fanés de splendeur. Ayant perdu « l'éclat ensorcelant de sa jeunesse, la dangereuse beauté de sa maturité ${ }^{23}$, il ne séduit plus toutes les femmes sans exception par sa simple entrée en scène. Sa verve s'applique surtout au récit de ses aventures passées, son esprit aux demandes de subsides.

Pourtant, au moment où le lecteur le rencontre sur une grand-route près de Mantoue, il semble avoir insensiblement fait les «corrections intérieures» qu'exige le vieillissement, s'être adapté ${ }^{4}$. Le dangereux libre penseur aux idées provocatrices édulcore peu à peu ses écrits; l'ex-favori des cours d'Europe, le conseiller des plus grands espère retrouver un poste à Venise, dès son retour ; l'amateur de femmes peut se flatter des œillades d'une jeune paysanne, des ardeurs amoureuses de son aubergiste, encore accorte.

C'est avant tout par le prestige de son passé que Casanova séduit encore, dans les salons provinciaux, la génération d'âge mûr; "l'énoncé de son nom suffisait encore, les derniers temps, à faire naître sur les lèvres des femmes l'expression d'une admiration tardive ou tout au moins un léger tressaillement du regard, aveu que l'on aurait beaucoup aimé le rencontrer quelques années plus tôt $»^{25}$.

Parfois Casanova oublie même son présent médiocre, son avenir misérable ; « rajeuni par son sourire », il se croit redevenu le Casanova d'autrefois! «Tandis qu'il racontait et fabulait ainsi, il lui semblait presque qu'il était encore vraiment aujourd'hui le Casanova gâté par la chance, insolent, éclatant, qui avait traversé la terre avec de belles femmes, que des princes du monde et de l'esprit avaient honoré des plus grandes 
faveurs, qui avait gaspillé, joué et donné des fortunes - et pas un pauvre hère déchu [...]; il oubliait même son but suprême, rentrer dans sa ville natale, qui l'avait méprisé et banni, pour y clore un destin jadis si flamboyant, comme le dernier de ses citoyens, comme un écrivassier, un mendiant, un moins que rien $! »^{26}$.

\section{Eros et Thanatos Arthur Schnitzler} son amant Lorenzi, sa pulsion d'amour, Casanova croit retrouver la plénitude de son être. N'est-il pas un dieu? L'extase abolit le temps, rend ridicules les vanités des humains, dérisoires leurs désirs ${ }^{31}$.

dès qu'il s'endort, la pulsion d'amour et de puissance, démultipliée par le refoulement, l'entraîne dans un rêve de démesure, où il réalise le "couronnement de l'œuvre de sa vie » : amour et patrie, Marcolina et Venise ! Grâce à " l'immense pouvoir de son être inextinguible » il fait sienne pour toujours Marcolina ; il rentre avec elle à Venise; son seul nom inspire l'effroi à tous les sénateurs; au jeu, il gagne, leur or d'abord, puis "leurs fortunes, leurs palais, leurs manteaux de pourpre", et les voit, devenus mendiants, ramper à ses pieds! S'élançant à la recherche de Marcolina, il glisse en gondole sur les eaux, puis dans une course toujours plus légère nage jusqu'à Murano et découvre enfin dans sa hâte qu'il vole, qu'il sait voler ${ }^{32} ! .$. Jeunesse, amour, richesse, puissance, pouvoirs surnaturels, éternité : quand son orgueil, sa paranoïa ne 
sont pas jugulés par la conscience ou l'intellect, Casanova est, en rêve, un Hermès triomphant.

Le rêve - hélas ! - tournera au cauchemar. Casanova y perdra tout: le pouvoir, l'or, Marcolina... et Venise, et mourra noyé en pleine mer. L'obsession du retour se révèle de façon prémonitoire - pulsion de mort.

\section{Venise aux miroirs : le trouble du temps Hugo von Hofmannsthal} un miroir sans indulgence. Pour la première fois, semble-t-il, et malgré les dénégations de son valet Le Duc l'assurant qu'il est plus jeune que tous les hommes de son âge, il prend ici conscience du temps qui passe. Ailleurs, régénéré par la nouveauté de chaque lieu, de chaque aventure, il use du temps qui semble ne pouvoir l'user. Ici il éprouve le besoin de faire de la culture physique, de soigner ses mains ${ }^{35}$. Il apprend que la très belle femme du Procurateur Manin est morte... depuis sept ans. Son passé vénitien l'entraîne à prononcer des mots comme "souvenir ", " de mon temps ", petite formule fatale à qui se veut jeune ${ }^{36}$ ! Symboliquement, les couleurs qui l'entourent, celles qu'il porte, sont un peu éteintes : meubles aux «dorures passées » chez lui, alors que chez Vittoria tout brille de fleurs fraîches et d'ors clairs ; costume " jaune très pâle et lilas ", alors que son portrait sur la tabatière - trente ans plus tôt - resplendit d'or et de saphirs.

Vittoria, la cantatrice, lui tend le miroir de la jeunesse préservée. Pour elle le fil du temps s'est rompu lorsqu'elle a été abandonnée ${ }^{37}$. Vivant hors du temps, elle n'a pas pu vieillir ; à trente-deux ans elle en avoue vingt-quatre et fait passer Cesarino son fils pour son jeune frère de seize ans. Son identité est l'amour, la fidélité à cet amour et à ses conséquences, les "présents de la vie", sa musique, son fils. Weidenstamm, lui, change constamment de vêtements, de nom, d'apparence ${ }^{38}$. Vittoria lui fait remarquer combien il est différent de son image d'autrefois, même si, à l'instant de la rencontre, 
l'ouragan de son sang lui a immédiatement révélé la présence du bien-aimés ${ }^{39}$. Elle lui fait le reproche de n'être pas même fidèle à son nom ${ }^{40}$. Peu importent le nom, l'habit, prétend Weidenstamm, puisque l'individu est bien le même. Paradoxe de l'aventurier ! Revenant incognito, déguisé, il aspire néanmoins à être reconnu. S'identifiant à Ulysse, ancêtre mythique, il a cette plainte ${ }^{41}$ :

Et Ulysse c'est à peine si son chien le reconnut !

Lui voudrait être reconnu, alors que son identité est fluctuante - « est-ce bien toi ?", lui demande Vittoria -. Il vit dans la répétition, non dans la durée, il n'a pas d'histoire, seulement des aventures, pas de mémoire, seulement des souvenirs. Il incarne la problématique du temps, que Hofmannsthal définit comme « antinomie de l'être et du devenir ».

L'aventurier n'a d'attache ni dans l'espace, ni dans le temps. On ne connaît ni son grand-père, ni sa grand-mère. Il n'a jamais connu son père, et peut-être a-t-il pour fils ce boiteux rencontré dans un village traversé il y a vingt ans. Devant son portrait sur la tabatière, il dit, parlant de sa propre image à la troisième personne ${ }^{42}$ :

Celui-là c'est mon père, car chaque jour est le fils du jour d'hier.

Il n'est donc pas un être en devenir dans le temps, mais une suite d'individus qui s'engendreraient l'un l'autre.

\section{Carpe diem Hugo von Hofmannsthal}

L'aventurier refuse donc sa propre chronologie, vit le temps de l'instant et non celui de la durée, car le temps qui passe n'est que «le silencieux valet ${ }^{43}$ de Celle qu'il ne veut pas nommer, la Mort. Mais à Venise il est confronté à cette chronologie, car « la vie met à chacun des doubles sur sa route $\aleph^{44}$. Lui qui se voudrait acteur de sa vie, et non spectateur - encore moins juge -, surprend sa propre image dans deux miroirs; le vieux compositeur et Cesarino sont à la fois lui-même, et, peut-être, son père, et son fils.

Le vieux compositeur Passionei, aujourd'hui retombé en enfance, a été autrefois très beau, très aimé des femmes, très célèbre. Il ne reste de lui que sa musique, qu'il ne reconnaît plus. Le baron voit en lui son propre vieillissement, inexorable : "voilà ce qu'il advient de nous! »... Et retourne à sa philosophie du carpe diem ${ }^{45}$.

- Cesarino est l'autre miroir où une étape de sa vie est enclose. Sans même avoir échangé un mot avec lui, le baron le reconnaît : c'est un autre lui-même, celui de la tabatière, d'il y a trente ans ${ }^{46}$ ! Après avoir bavardé avec lui, en le tutoyant - comme se tutoient des amis, dit-il, comme se tutoient père et fils, le reprend Cesarino, qui continuera à ignorer que Weidenstamm est son père -, il sait que Cesarino a tous les dons, toutes les aspirations qui étaient, qui sont encore les siens. Et quand il lui dit, pour tout adieu: « va, va, mon fils, ô comme je te reconnais », il salue en lui un fils spirituel et adresse un regard nostalgique à sa propre jeunesse.

En se penchant un instant sur le miroir d'hier et sur celui de demain, l'aventurier éprouve, de façon quasi physique, la menace du temps. Il a choisi de courir après chaque bulle de savon, préféré les masques fardés à la beauté d'un cœur fidèle, a toujours voulu tout étreindre : il a été «l'esclave de la vie et n'a pas su vieillir » ${ }^{47}$. Le 
bilan est lucide. Mais fidèle à lui-même, l'aventurier ne pourra que repartir vers sa solitude et sa quête de l'instant.

Vittoria! Tais-toi, Vittoria!

Nous devons nous croiser en silence, muets

comme les deux seaux qui se croisent dans le puits ;

l'un monte, celui qui est plein,

l'autre, le seau vide, s'enfonce dans l'obscuritét8.

Constat doux-amer.

\section{La marche obsédante du temps Arthur Schnitzler} une des dimensions de l'être. Certes, lui aussi, vit avant tout intensément dans l'instant; il a conservé l'étonnante capacité de réagir sur-le-champ à toute situation nouvelle et tout le récit est une succession de ses retournements agiles, avec mimiques, monologues intérieurs appropriés. Mais il a en même temps le sens de la chronologie. Casanova vit en champion de l'instant, mais porteur de toute une expérience, de toute une légende. Weidenstamm se veut sans histoire, sans racines ; Casanova est ancré dans un passé personnel, littéraire, politique. Il évoque avec plaisir son enfance à Venise, sa mère, ses protecteurs, ses premières aventures; il est au courant des mouvements scientifiques, littéraires et philosophiques de son temps, y joue même sa partie ; il suit l'évolution politique de l'Europe, de Venise. Une des constantes de la nouvelle est la trace laissée par le passé dans le présent, lourde de symboles ${ }^{49}$.

Les paroles de Casanova, ses monologues, font souvent référence au passé, en un perpétuel balancement entre le plaisir de J'évocation et la tristesse, ou la colère, que suscite la comparaison entre le Casanova d'autrefois et celui d'aujourd'hui.

Le parallèle est amer en maint domaine - financier, intellectuel, social -, mais, bien sûr, pour cet homme mûr, qui fut très séduisant, qui veut conquérir une toute jeune femme, la dégradation physique est ressentie comme particulièrement douloureuse. Dans le regard des autres - même si certains ont l'indulgence de l'amour - Casanova voit une image de vieil homme; lucide, il se peint lui-même avec un réalisme grinçant ${ }^{50}$; ironique, il fait des grimaces à son reflet dans la glace ! Et dans les yeux de Marcolina, après la nuit d'amour volée, ce n'est pas le dégoût devant la duperie qu'il lira, mais l'injure suprême, parce qu'irréversible: l'horreur qu'inspire ce "vieillard»" ${ }^{51}$ La dégradation physique est la preuve sensible d'une décadence. Ille constate : envolé son pouvoir sur autrui, tari l'effet de sa seule personne! Son temps est « fini»! Et ce mot " fini » devient lancinant battement à ses tempes, martellement de déchéance et de révolte ${ }^{52}$. En face du jeune et beau Lorenzi, reconnaissant en lui son double d'autrefois, il est contraint de s'avouer $»^{53}:$ « que me reste-t-il du Casanova qui était jeune, beau et heureux ? Ne suis-je pas mort depuis longtemps?»

Casanova ne vit plus chaque aventure comme une parcelle d'éternité renouvelable à loisir. Vieillir lui apprend la vanité de bien des choses: à quoi bon écrire contre Voltaire, pourquoi perdre du temps pour une petite aventure avec une jeune paysanne ou pour un détour chez son ami Olivo? Le leitmotiv de son "voyage de retour " est l'impatience. Cette hâte exacerbe les pulsions essentielles. Elle est un de ces «états d'âme nouveaux ", exceptionnels, capables de bouleverser le " naturel » de l'individu, 
comme l'a montré Schnitzler dans son étude sur les dons et états d'âme ${ }^{54}$. Elle entrâne Casanova à agir de façon non conforme à sa nature, le contraint, pour satisfaire sa double passion, à la lâchetée ${ }^{5}$, l'infidélité à lui-même.

\section{Le reniement : une mort symbolique Arthur Schnitzler}

Casanova possédera Marcolina, rentrera à Venise, mais au prix d'un triple reniement. Reniement intellectuel : après s'être assuré l'oreille attentive du Conseil des Dix en renonçant à sa réputation de libertin et de libre penseur et en s'opposant à «l'hérétique» Voltaire, il accepte, pour rentrer au plus vite, le marché de Bragadin : espionner pour le compte de ce gouvernement d'inquisition «les éléments libres penseurs, irréligieux et dévergondés » de Venise.

Reniement moral: il abandonne son code éthique habituel; lui, le prince du libre arbitre, se fait acheter pour espionner ses frères en esprit. Lui qui ne regrettait jamais ses largesses, accepte de récupérer la dot autrefois donnée, parce qu'il pressent que cet argent va lui permettre un odieux marché : acheter à Lorenzi, son rival amoureux, une nuit avec Marcolina. Son tempérament de joueur prodigue s'est fait calculateur, intéressé, mesquin.

Reniement amoureux enfin. Casanova, dont l'essence même était jusqu'alors la séduction, ne parvenant pas à charmer Marcolina, l'achète tout simplement à son amant Lorenzi, donnant à ce dernier de quoi payer une dette de jeu, lui épargnant le déshonneur et le suicide. La nuit de félicité est, puisqu'il se fait aimer en se faisant passer pour Lorenzi, une nuit de mensonge, qui déshonore cette femme et la féminité, « une faute inexpiable de la ruse contre la confiance, du plaisir contre l'amour, de la vieillesse contre la jeunesse ${ }^{56}$.

Par ce triple reniement Casanova renonce à lui-même plutôt qu'à ses désirs, conformes à sa personnalité, mais rendus obsessionnels par la contrainte du temps. L'impatience qui l'habite le fait par deux fois - au moment décisif - écarter un sursaut d'honnêteté, de fidélité à lui-même. S'apprêtant à passer la nuit chez Marcolina sous l'identité de Lorenzi, il se demande s'il était bien indispensable de commettre cette vilenie. « $\mathrm{Ne}$ serait-on parvenu au même but, avec un peu de patience ?... cinq jours... trois jours - et elle aurait été mienne - sciemment mienne [...] Je pourrais revenir, - demain, aprèsdemain... et commencerais mon entreprise de séduction - pour ainsi dire en honnête homme $\wedge^{57}$. Mais le temps presse... et le désir de Venise.

De la même façon, quand il reçoit la lettre où Bragadin l'incite à servir le Conseil comme espion, "par amour de la patrie ", - et contre salaire - il a tout d'abord une violente réaction de colère, tremble de chaud et de froid, et décide de refuser l'humiliation de ces gredins qui croient pouvoir faire de lui un mercenaire. Enflammé de dégoût et de honte, il forge en un instant le plan orgueilleux d'une vengeance raffinée ${ }^{58}$, que son intelligence préservée, son énergie retrouvée lui feront mener à bien. Il rentrera incognito à Venise et prendra tout son temps pour fomenter la révolte contre l'odieux gouvernement des hommes ; il bravera le Dieu impie des cieux, auquel il croit moins que jamais, ce Dieu qui n'a pas su reconnaître ses mérites, et... il « réglera ses comptes à ce petit écrivaillon de Voltaire ». Hélas! L'émissaire impatient attend la 
réponse. Se révolter serait un luxe de jeune homme: Casanova, ravalant son dernier sursaut de fierté, rédige la réponse attendue à Venise, flatteuse et servile.

Il cesse en cet instant d'être Casanova. Les deux journées passées chez Olivo ont été la dernière enclave sur le chemin du retour: univers clos dans le temps et l'espace, lieu tragique, où l'ultime face à face du héros avec lui-même a pour dénouement sa mort symbolique et son départ pour le dernier voyage.

\section{Retour interrompu et mythe pérenne Hugo von Hofmannsthal}

53 Le constat de retour est ambigu, comme en étaient ambigus les motifs. Certes, Venise, objet de sa nostalgie, ne déçoit pas l'aventurier : immuable, elle est sa seule fidélité. La journée de retrouvailles est « ravissante » : une œuvre d'art née de l'imagination d'un poète. Pourtant l'aventurier sent s'incruster en lui les brûlures au fer rouge que lui imprime le passé. Redoublement et dédoublement imposés par le destin mènent à cet aveu : « je me rends compte qu'on ne doit pas vouloir vivre deux fois la même chose " . L'aventurier qui revient court le risque de se répéter, donc de perdre son identité. Weidenstamm, son trouble à peine avoué, décidera, sans transition, d'une pirouette, de repartir.

Et pourtant Venise restera - à tout jamais, aimerait dire Hofmannsthal - patrie idéale de ce Casanova qui semble en être l'émanation, et terre d'élection du mythe de l'aventurier. Cesarino $0^{60}$ perpétuera le mythe en le perfectionnant : la première partie de la pièce vibre de tout l'éclat de Weidenstamm; le second versant sera illuminé du feu du nouveau Casanova.

Cesarino est le portrait vivant du jeune homme peint sur la tabatière ${ }^{61}$. Il a déjà toutes les caractéristiques du séducteur. Il est le double de son père, mais magnifié. Son charme est quelque peu magique, son art de la conquête presque magnétique. Il est limpide et lisse comme l'eau, léger comme l'air ; le feu de son regard, la flamme de ses paroles ont l'innocente impétuosité de la jeunesse... Wiedenstamm se targue de l'agilité d'Hermès. Cesarino ne serait-il pas tout à la fois Hermès (célérité, goût du voyage, de l'or et des trésors), Cupidon-Adonis (ses yeux qui « débusquent l'amour », son sens de la beauté), Apollon enfin? Il en porte les couleurs - or et saphir-, il est comme lui familier des dauphins et surtout « fait de tout ce qu'il touche de la musique ». L'univers est pour lui une unité magique, cette "harmonie des sphères ${ }^{62}$ que la musique exprime parfaitement, alors que les paroles sont, selon lui, des harpies qui jettent un linceul sinistre sur la beauté de la vie, Etre plus tard semblable à ce baron inconnu qui lui plaît tant ? Bien sûr, mais avec, en plus, célébrité... et humour, puisqu'il porterait cette célébrité non comme une perruque officielle, mais comme un simple bijou ${ }^{63}$. Comme le remarque Hofmannsthal, «Cesarino est en face de Weidenstamm un double à la fois critique et accompli, idéal $»^{64}$. Discrètement il le dote d'attributs qui sont traditionnellement médiatiques entre les éléments de la cosmogonie, le dauphin, le cyprès, le corail -, et symboles d'éternité.

Weidenstamm reconnaît en Cesarino son fils et son successeur, Mais il repartira sans s'être fait connaître. Cesarino à son tour est un aventurier sans racines, ignorant la véritable identité de ses deux parents. Pourtant, en abandonnant son "destin » de père ${ }^{65}$, Weidenstamm l'intègre de façon symbolique à la lignée des aventuriers de 
Venise. Il voulait, en offrant à Lorenzo Venier une tabatière avec son portrait adolescent (Vernier y reconnaît Cesarino et comprend que Weidenstamm est son père), sceller leur amitié : c'était en même temps lui léguer par avance le rôle de père. Le dernier regroupement des protagonistes montre, en face du baron seul, Lorenzo entouré de Cesarino et Vittoria. La dernière phrase de Weidenstamm à Cesarino est celle d'un père qui coupe les liens: «va, va, mon fils!», alors que Lorenzo, deux répliques plus loin, lui dira, avant de quitter la scène avec lui, « Cesarino, viens ! ».

En offrant à Cesarino une bourse, un autre attribut d'Hermès, et un anneau gravé, Weidenstamm fait de lui son héritier.

Après quelques heures de désordre intérieur, une minute d'embarras au départ de Cesarino, Weidenstamm retrouve son attitude d'aventurier qui sait l'art de partir sans se retourner ${ }^{66}$. Il reprend la route. Il a pour Vittoria remis le temps en marche. Il rétablit l'unité de son être dans l'amour et la musique (elle chante le grand air de Bacchus et Ariane). Et il laisse à Venise, devant tout le champ du possible, l'aventurier Cesarino, qui perpétuera le personnage de Casanova, un des porteurs de ce que Hofmannsthal appelait la "mythologie européenne ", et dont il a avec tant de vigilance enregistré les dernières heures glorieuses.

\section{Retour parodique et mythe assassiné Arthur Schnitzler}

En se reniant le vieil aventurier de Schnitzler a perdu son identité de Casanova. Il avait pu s'imaginer un moment, en rêve, avoir trouvé, en usurpant l'identité de Lorenzi, une jeunesse nouvelle, et un amour idéal, gages d'éternité. Mais au petit matin il n'est plus qu'un vieillard ridicule, décidé, après cette dernière aventure peu glorieuse, à reprendre la route de Venise. Sa tragédie personnelle, initiation à rebours, - devenir un vieillard quelconque après avoir été Casanova-, semble consommée. Pourtant Schnitzler fait mourir symboliquement son héros une seconde fois en le faisant meurtrier de son propre double et héritier spirituel, Lorenzi. Automutilation par le reniement, puis castration du fils.

La simple évocation de la nièce d'Oliva, Marcolina, belle, intelligente et vertueuse, avait enflammé Casanova d'un désir capable de concurrencer le désir de Venise. Voulant assouvir cette pulsion, il se trouve confronté au personnage de Lorenzi, fringant lieutenant permissionnaire, séducteur peu scrupuleux, familier de la maison d'oliva, qui est, - Casanova n'en doute pas un instant et le hasard d'une promenade nocturne le lui confirmera -, l'heureux amant de Marcolina. Dès la première rencontre, d'un seul regard, ils ont pris la mesure l'un de l'autre ; la jalousie s'installe ; Lorenzi se montrera blessant, Casanova ironique. Casanova se demande un instant qui ce Lorenzi lui rappelle. «C'est son propre portrait qui, rajeuni de trente ans » lui fait face. Se serait-il « réincarné » en Lorenzi ? Pour « revenir », il faudrait d'abord être mort, songe-t-il! Mais sa vie de vieil homme n'est-elle pas une forme de mort ${ }^{67}$ ? Lorenzi et Casanova sont interchangeables dans le rêve tant ils sont semblables dans la réalité. En rêve, Amalia, l'épouse d'oliva, autrefois maîtresse de Casanova, voit un Casanova jeune et triomphant rentrer à Venise, poursuivi par un Lorenzi pauvre et vieux, qui lui demande l'aumône ${ }^{68}$ ! Dans la vie ils ont en commun l'apparence physique, âge mis à part, - taille élancée, traits marquants, et celle lueur singulière au fond des yeux - ; l'attitude face au jeu ; le comportement avec les femmes; le goût du risque ; l'audace ; une « morale » qui 
fait fi de toute pusillanimité. "Nous sommes faits du même bois, Lorenzi, sommes frères en esprit, et nos âmes peuvent donc s'affronter sans fausse pudeur, fières et nues!»C'est avec ces mots d'introduction ${ }^{69}$ que Casanova propose à Lorenzi le marché qui lui assurera la possession de Marcolina, en permettant à Lorenzi d'échapper au déshonneur. Chacun possède ce que l'autre désire ardemment. Ils concluront le troc pièces d'or contre Marcolina - lors d'un saisissant face à face : ils échangent, sans une parole, les ducats contre le manteau bleu nuit d'officier : symbolique du manteau que Lorenzi laisse tomber à ses pieds ${ }^{70}$, se dépouillant d'une identité que Casanova, devenant ainsi son propre double, revêtira pour s'approprier Marcolina.

Le premier rai de lumière ayant révélé à Marcolina la véritable identité de son amant, Casanova s'enfuit sous le regard d'horreur de la jeune femme, qui prend conscience du marché. Enveloppé du seul manteau bleu de l'officier, il referme la porte du domaine. Mais en travers de la route du retour vers Venise surgit, flamboyant de rouge et d'argent, Lorenzi qui, pour laver son déshonneur, demande raison dans un duel sans merci. Dans un dernier sursaut de lucidité Casanova constate qu'il ne lui en coûtera pas de perdre les quelques années vaines qui lui restaient à vivre. Lorenzi se dresse devant lui, prêt au combat, et, pour être à égalité avec son rival nu sous le manteau bleu, il paraît "dans la superbe nudité d'un jeune dieu " ${ }^{71}$. La tentation de jeter son arme, d'étreindre ce rival fraternel effleure Casanova. L'ardeur du combat leur donne la dimension de dieux antiques. "Jeunesse, vieillesse, fable que tout cela, pensait-il... Ne suis-je pas un dieu? Lui et moi des dieux? $»^{72}$. Blessé au cœur, Lorenzi s'effondre. Casanova sent que la meilleure partie de lui-même gît à ses pieds, dans "l'inégalable beauté de la jeunesse », et l'embrasse sur le front en murmurant : «Bienheureux ! ».

En prenant l'identité de Lorenzi Casanova n'a pas retrouvé la jeunesse. Meurtrier de son double, il a éliminé son possible successeur. Le mythique Casanova meurt sans descendance.

Le vieil aventurier qui, en quarante-huit heures, a mis lui-même fin à son destin de Casanova, reprend alors, en une fuite vertigineuse, rythmée par les seuls changements de poste, la route de Venise. Il s'enfonce pour les deux jours et deux nuits de voyage «dans le plus profond sommeil de sa vie». Dans la confusion de ce sommeil réalité rêvée et réalité vécue sont indissociables et l'aventurier, allant jusqu'au bout de la perte d'identité, doute de son existence même. "Parfois il n'était pas Casanova, mais Lorenzi, pas le vainqueur, mais le vaincu, pas le fuyard, mais le mort, dont seul le vent du matin caressait le corps pâle du jeune homme ; et tous deux, lui et Lorenzi, n'avaient pas plus de réalité que les sénateurs aux manteaux de pourpre rouge (de son rêve) $»^{73}$.

$\mathrm{Au}$ matin du troisième jour il atteint enfin Mestre puis Venise. Bon nombre des récits de Schnitzler trouvent leur dénouement à l'aube, symbole de renouveau. Pour l'aventurier, le jour gris qui se lève sur une Venise grise est une aube ironique, sans espoir ni désespoir, qui éveille quelques souvenirs, mais nul projet. Le voyage s'achève dans une pauvre chambre d'auberge, " une pièce morne avec vue sur le mur aveugle de la maison d'en face $\aleph^{74}$. Retour sans retrouvailles: l'aventurier passe sa première journée à organiser sa stratégie de survie : il rend visite à son employeur Bragadin, brûle ses vieux papiers, commence son métier d'espion. Il a encore un certain nombre des tics de Casanova. Il a conservé son insatiable faim; il dévore avec une «bestiale avidité » le repas Proposé par Bragadin - substitut d'action ${ }^{75}$; il divertit la société du café Quadri, où il vient espionner, avec plus d'impertinence que jamais - il lui faudra dorénavant vivre des intrigues d'autrui, par procuration. Il rode le personnage qu'il 
jouera désormais, «charmant et peu dangereux $»^{76}$. Se parodiant lui-même il n'est plus qu'un automate qui retrouve les itinéraires d'autrefois «avec l'assurance d'un somnambule ». Son retour à Venise est marqué par l'extinction des deux expressions de la vie intérieure, caractéristiques des personnages de Schnitzler, que sont le monologue intérieur et le rêve. Le sommeil qui, lourd et sans rêve, finit par avoir pitié du vieil aventurier, annonce le dernier sommeil qu'il est venu chercher dans cette ville où «sous les ponts coulent les canaux noirs qui vont vers les eaux éternelles".

Le retour de l'aventurier met à nu sa solitude. Le Casanova de Hofmannsthal, en prenant la mesure, l'assume avec panache : elle est la rançon du voyage, la gloire du voyageur. Le Casanova de Schnitzler, las de l'errance, se fait, pour retrouver une patrie indifférente, déserteur de lui-même, assassin du mythe. Pessimisme d'un Schnitzler vieillissant et plongé dans les troubles de la guerre ? Sans doute... À la même époque, dans Casanova à Spa ${ }^{77}$, il donne pourtant la parole à un tout autre Casanova, qui, fort de la vitalité de ses trente-deux ans, affirme que l'homme libre n'a d'autre patrie que son cœur, proclame la vanité de tout retour et s'écrie :

Partir ! - Pour l'âme un éternel appel !

\section{NOTES}

1. Hugo von Hofmannsthal, Der Abenteurer und die Sangerin oder die Geschenke des Lebens, Dramatisches Gedicht in einem Aufzug (mit einer VerwandJung). La pièce fut conçue et écrite lors d'un séjour à Venise (21.IX.1898 - 10.X.1898), reprise (janvier-mars 99), modifiée jusque fin 99, en tenant compte en particulier des remarques de Schnitzler sur le découpage de la pièce. Première publication en avril 99 dans Die Neuedeutsche Rundschau, S. Fischer Verlag, Berlin; première représentation simultanément au Deutsches Theater de Berlin et au Burgtheater de Vienne le 18 mars 1899. Dans une lettre à son ami Bodenhausen (5. V.1903) Hofmannsthal écrit, quelques années plus tard, qu'il aime énormément cette pièce et ajoute : «Ich glaube, es enthält mehr, als man beim ersten Lesen denkt, nicht in den Einzelheiten, sondern im Bezug des Ganzen, in der Philosophie, die es hat».

2. Arthur Schnitzler, Casanovas Heimfahrt. Première publication dans Die Neue Rundschau, juilletseptembre 1918. Dans les années 1914-1916 Schnitzler lit avec grand plaisir les Mémoires de Casanova. Il travaille parallèlement à sa pièce Les sœurs ou Casanova à Spa, qui paraîtra en 1919.

3. Hugo von Hofmannsthal, Der Abenteurer und die Sängerin, dans Theater in Versen, S. Fischer Verlag, Berlin, 1905, pp. 127-260. Personenregister, p. 131.

4. Weidenstamm est une des nombreuses variations de Hofmannsthal sur le personnage de Casanova, et celui de l'Aventurier. L'Aventurier est pour lui une figure "mythique ", plutôt positive comme l'indiquent les nombreuses réflexions - dont beaucoup concernent nommément l'aventurier Weidenstamm-Casanova - de Ad me ipsum, Aufzeichnungen, S. Fischer Verlag, Berlin, 1973, p. 213-244.

«Der Abenteurer eine mythische Figur. Desgleichen Ariadne» (p. 240) - «Der Abenteurer, jener die Totalitat umfassende, umarmende Geist» (p. 221) - «Auserlesenheit / Kaiser - Abenteurer - 
Zauberer - Weiser - Dichter - Kind - Wahnsinniger.

Geistige Souveränität : sieht die Welt von oben

Nachteil: sieht nur Totalität (...)

Gabe sich zu verfältigen: die Spiegelungen» (pp. 214-215).

Voir sur ce sujet : Ewald Grether, «Die Abenteurergestalt bei Hugo von Hofmannsthal», Euphorion, 48, 1954. Et William Rey, «Dichter und Abenteurer bei Hugo von Hofmannsthal», Euphorion, 49, 1955.

5. Op. cit., pp. 147-148:

Ich achte diese Welt nach ihrem Wert,

ein Ding, auf das ich mich mit sieben Sinnen

so lange werfen soll, als [...]

atmen! wie ein Schwamm

die Welt einsaugen.

6. Weidenstamm dit avoir en mémoire les mensurations de dix-mille femmes, mais préférer les parer de vêtements plutôt que les sculpter dans le marbre : «ich habe nicht die Laune, mich mit totem Material abzugeben», Idem., p. 157.

7. Ibid., p. 144 :

Dann ist's die Arche Noah! Jeder Art

ein Tier. Und dass so viele Arten sind,

das macht die Welt so bunt.

8. Ibid., p. 164 :

Ihr lacht! Den Teufel, ja, den spiel ich gern,

den meint Ihr doch, Abbate, der den grossen

Goldklumpen nachts ins Netz des armen Fischers warf?

p. 178 :

Und Zufall tanzt der übermütige Gott,

wie ein betrunkener Stern in dunkler Luft

und streut Verwirrung! Doch ich nehm's auf mich!

9. Ibid., p. 246 :

Also weisst Du nicht zu schenken,

da du sogar nicht anzunehmen weisst!

10. Comme le mot Lust, le mot Augenblick revient à chaque instant dans les propos du baron, p.

ex., ibid., p. 243 :

dort lernst Du's, jeden kurzen Augenblick

so leer zu saugen, wie ein Bettelkind,

das Trauben stahl, die letzte Beere aussaugt.

Und das ist gut, denn keiner kommt zurück.

11. Ibid., p. 243 :

Der umgegrabne Baum geht schnell zugrund, uns gibt ein fremder Boden Riesenkräfte.

12. Sur la parenté Casanova / Hermès, voir Erwin Kobel, Hugo von Hofmannsthal, Walter de Gruyter, Berlin, 1970, p.96. Sur la présence de Hermès à Venise comme constante de la littérature, voir Walter Pabst, «Satan und die alten Götter in Venedig», Euphorion, 49, 1955.

13. Op. cit., p. 202 :

Und sic schreibt, sic wisse,

was mich veranlasst hat, hierherzugehen.

Ich weiss es selbst nicht! ausser Uebermut, der Mäuse immer wieder zu der Falle hinlockt. 
14. Idem., p. 145 :

Rückkehren

und nicht vergessen sein: der Mund wic Rosen

die offnen Arme da, hineinzufliegen!

Als wär man einen Tag nur fern gewesen -.

15. Ibid., p. 139 : «die glücklichsten Stunden, dit unbeschreiblichsten, die unvergesslichsten...».

16. Ibid., pp. 242-243 :

Europa wird Dein Haus, die WeIt Dein Garten, der Wunsch erschafft Dir Vaterländer.

17. Ibid., p. 133 : «Ihr heisst Venier, ich heisse Weidenstamm. Ihr gehört zu den Familien, die diese Stadt regieren, ïch liebe diese Stadt über alles!».

18. Ibid., p. 136 :

Du bist ein Venezianer, ich bin's zehnfach!

der Fischer hat sein Netz, und der Patricier

das rothe Kleid und einen Stuhl im Rat,

der Beller seinen Sitz am Rand der Saule,

die Tänzerin ihr Haus, der alte Doge

den Ehehring des Meeres, der Gefang'ne

in seiner Zelle früh den salzigen Duft

und blassen Wiederschein der Purpursonne:

ich schmecke alles dies mit einer Zunge!

19. Ibid., p. 137 :

Welch eine Luft ist das! In solcher Nacht

ward diese Stadt gegründet. Ihre Augen

schwammen in Lust, er hing an ihrem Hals,

sie tranken nichts als aufgelöste Perlen.

[...]

Doch später dann zerging die Zauberstadt -

nicht ganz! es blieb ein Etwas in der Luft,

im Blut! Mit rosenfarbenen Muschellippen küsste

das Meer und leckte mit smaragdnen Zungen

die Füsse dieser Stadt!

20. Longue évocation lyrique de ces fêtes, ibid., pp. 145-146, ou La culture de la Renaissance en Italie de Burckhardt (1860) mise en vers !

21. Ibid., p. 139 : «Sie haben die Beredsamkeit eines Dichters, mein Baron - Oh, eines Liebhabers, höchstens eines Liebhabers».

22. Ibid., p. 203 :

O schöne Stadt,

die nie versagt!

23. Arthur Schnitzler, Die erzählenden Schriften, S. Fischer Verlag, 1961, II, Casanovas Heimfahrt, pp. 231-323. P. 231 : «Als Casanova langst nicht mehr von der Abenteuerlust der Jugend, sondern von der Ruhelosigkeit nahenden Alters durch die Welt gejagt wurde [...]».

24. Idem., p. 242 : «im berückenden Glanz seiner Jugend oder in der gefahrlichen Schönheit seiner Mannesjahre».

25. Arthur Schnitzler, Tagebuch 1913-1916, Wien, 1983, p. 296 : «Innere Correcturen [...] ; sonst könnte man nicht leben».

26. Op. cit., p. 243 : «er vergass sogar, dass es ihm wie ein höchstes Ziel erschien, in der Vaterstadt, die ihn eingekerkert und nach seiner Flucht geächtet und verbannt hätte, als der geringste ihrer Bürger, als ein Schreiber, als ein Bettler, als ein Nichts - sein einst sa prangendes Dasein zu beschliessen». 
27. Pour Schnitzler comme pour Hofmannsthal, l'Aventurier fait partie des archétypes humains, mais il est évalué différemment. Dans son essai Der Geist im Wort und der Geist in der Tat (dans Arthur Schnitzler, Aphorismen und Betrachtungen, S. Fischer Verlag, 1967) et les diagrammes joints, l'Aventurier est classé parmi les figures négatives. Mais le personnage de Casanova, tel que le peint Schnitzler, ne saurait être réduit à une illustration littéraire "symétrique » de l'essai, analysée par Dieter Hornig, "La stratégie narrative d'Arthur Schnitzler ", Actes du congrès Schnitzler, 1981. Publications de l'Université de Rouen, pp. 79-97, La nouvelle de Schnitzler, publiée dans le cycle «Die Alternden», est une réflexion sur l'âge, la solitude, l'écriture, le jeu, etc... (voir infra, note 55).

28. Op. cit., p. 231 : «er fühlte in seiner Seele das Heimweh nach seiner Vaterstadt Venedig so heftig anwachsen, dass er sie, gleich einem Vogel, der aus luftigen Höhen zum Sterben allmählich nach abwärts steigt, in eng und immer enger werdenden Kreisen zu umziehen begann».

29. Idem., p. 232 : «Denn er zweifelte nicht, dass er sich sofort besser befinden würde, wenn er nur erst der ersehnten Heimat wieder um einige Meilen näher gerückt wäre».

30. Ibid., p. 231 : «ein schmerzliches Sehnen »; p. 235 : «seine Ungeduld überkam ihn mit neuer Macht»; p. 268 : «Die Stadt seiner Jugend stieg vor ihm auf, umflossen von allem Zauber der Erinnerung, und das Herz schwoll ihm in einer Sehnsucht, so qualvoll und über alles Mass, wie er sie noch nie empfunden zu haben glaubte. Auf die Heimkehr zu verzichten erschien ihm als das unmöglichste von allen Opfern»; p. 249 : «Ich bettle bei den Hohen Herren in Venedig um ein Amt, um ein Stück Brot, um Heimat».

31. Ibid., p. 307 : «War an diesen Lippen nicht Leben und Sterben, Zeit und Ewigkeit eines? War er nicht ein Gott - ? Jugend und Alter nur eine Fabel, von Menschen erfunden? - Heimat und Fremde, Glanz und Elend, Ruhm und Vergessensein - wesenlose Unterscheidungen zum Gebrauch von Ruhelosen, von Einsamen, von Eiteln - und sinnlos geworden, wenn man Casanova war und Marcolina gefunden?».

32. Ibid., p. 309 : «Casanova ging, flog, flatterte hin und her [...] Hätte ich das früher gewusst, dass ich fliegen kann».

33. Hugo von Hofmannsthal, Aufzeichnungen, op. cit., p. 221 : «Der Abenteurer [...] in die Sphäre des Lebens gefallen: der Zeit und den verändernden Gewalten ausgeliefert».

34. Casanova, Mémoires, Édition de la Pléiade, Gallimard, Paris, 1959, t. II, ch. XXIX, pp. 696-713.

35. Au soir du premier jour passé à Venise, Weidenstamm, ressentant un manque de souplesse dont il accuse le voyage ! - fait des exercices de lutte avec Le Duc et lui recommande par deux fois en quelques minutes de commander à Marseille la crème pour les mains.

36. Op. cit., p. 137 : «Zu meiner Zeit sass auch der Alte noch».

37. Idem., pp. 186-187 :

Mir ist, ich hab'in dieser Stadt,

wo keine Gärten sind, nur Stein und Wasser,

nicht altern können, nicht wie And're altern. [...]

Du warst mir Frühling, Sommer, Sonn' und Mond

in einem!

38. Racontant sa fuite des Plombs, Weidenstamm énumère les costumes superposés pour amortir sa chute. Cette insistance montre que pour lui les vêtements sont plus que simples déguisements, comme il l'explique à Cesarino (p. 245) :

Auch Kleider sind kein Ding, ganz zu verachten,

nichts ist bloss äusserlich. [...]

In diesen Dingen steckt ein Theil von uns.

39. Ibid., p. 186 :

Sonderbar,

jetzt seh'ich Dich verändert, im Theater 
war's wie ein Blitz, bei dem mein Blut im Sturm

Dein früh'res Bild auswarf.

40. Ibid., p. 186 : Auch Deinen Namen trägst Du nicht mehr, hast wie ein altes Kleid ihn abgelegt.

41. Ibid., p. 145 :

Und den Ulysses grüsste kaum sein Hund.

42. Ibid., p. 182 : Baron (zeigt auf sein Bild auf dem Deckel der Dose):

«Er ist mein Vater, denn ein jedes Heut' ist seines Gestern Shon».

43. Ibid., pp. 202-203 :

$\mathrm{Du}$,

den ich nicht nennen will, und dem die Zeit auf leisen Sohlen dient.

44. Ibid., p. 225 :

Vittoria : « Das Leben

schickt Jedem Doppelgänger über'n Weg»

45. Ibid., p. 239 :

Das wird aus uns!

[...] Er ist nicht doppelt

so alt wie ich, und wär'er's, war's kein Trost!

Nur keinen Tag verlieren, keiner kommt zurück!

46. Ibid., p. 234 :

Ein Spiegel ist's,

der dreissig Jahr nach rüekwärts, wie ich athme, mich eilig blitzt ! lch küsse meine Jugend wehmütig auf die Stirn, wenn ich ihn küsse!

47. Ibid., p. 256 :

(Einer), der mit tausend Armen

nach allen Freuden griff und wie ein Kind mit allem wild zum Mund fuhr; der mit Lust am Schein von Seifenblasen hing; der achtlos ein wundervolles Herz hinfallen liess, um eine liederlich geschminkte Maske zu haschen; der des Lebens Sklave hiess, nicht altern konnte.

48. Ibid., p. 256 :

Vittoria! still, Vittoria!

Wir müssen still vorüber aneinander, still wie die beiden Eimer in dem Brunnen, der eine geht nach oben, der ist voll, der leere geht nach unten in das Dunkel.

49. P. ex. Décors : le château, délabré au dehors, confortable à l'intérieur, le vieux couvent austère qui cache un cloitre vivant et parfumé. Destin : la dot autrefois offerte qui entraîne aujourd'hui la reconnaissance d'olivo, la réapparition des ducats. Fidélité : la voix de l'amante d'autrefois qui, rompant son vœu de religieuse cloîtrée, crie le nom de Casanova, lui faisant comprendre la vraie nature de l'amour...

50. Op. cit., p. 248 : «Die Runzeln meiner Stirn... Die Falten meines Halses! Und die tiefe Rinne da von den Augen den Schläfen zu! Und hier - ja, hier in der Ecke fehlt mir ein Zahn. Und diese Hände, Amalia Sieh sie doch an! Finger wie Krallen ... kleine gelbe Flecken auf den Nägeln ... Und die Adern da - blau und geschwollen - Greisenhände!». 
51. Idem., p. 217 : «Und dieses Wort "vorbei” hämmerte in seinen Schläfen weiter, als sei es bestimmt, von nun an der Pulsschlag seines verlorenen Daseins zu werden»

52. Ibid., p. 259 : «Bin ich denn nicht seit lange gestorben? Was ist denn noch an mir von dem Casanova, der jung, schön und glücklich war?».

53. Voir dans Der Gelst im Wort und der Geist in der Tat le chapitre intérieur intitulé «Ein Zwischenkapitel über Begabungen und Seelenzustande», qui apporte des nuances aux diagrammes, et l'analyse qu'en fait Françoise Derre, L'œuvre d'Arthur Schnitzler, Didier, Paris, 1966, p. 238.

54. La conduite infâme de Casanova ne fait pas naître chez le lecteur un sentiment d'horreur, comme l'ont justement constaté les critiques. Ceci n'est sans doute pas uniquement dû au recul historique, que fait remarquer Françoise Derré, mais aussi à la pitié qu'éprouve Schnitzler, et partant le lecteur, pour son héros, que l'on sent acculé par le temps aux pires turpitudes, qui peuvent aller jusqu'à une espèce d'auto-avilissement gratifiant, comme le montre l'épisode du viol de la petite Teresina, sorte de réponse à la lettre de Bragadin : renforcement de la déchéance. Sur cette pitié de Schnitzler pour son héros vieillissant, et leur parenté, voir Maurice Gode, «Le retour de Casanova », Cahiers d'Études germaniques, Université de Provence, 1984, pp. 99-122.

55. Op. cit., p. 311 : «als hätte in dieser Nacht List gegen Vertrauen, Lust gegen Liebe, Alter gegen Jugend sich namenlos und unsühnbar vergangen».

56. Idem., p. 306 : «Wäre man nicht mit etwas Geduld zum gleichen Ziel gekommen? [...] Fünf Tage ... drei - und sie hätte mir gehört -wissend mir gehört [...] Ich könnte wiederkommen, - morgen, übermorgen... und begänne das Werk der Verführung - als ehrlicher Mann sozusagen».

57. Ibid., pp. 287-288.

58. Op. cit., p. 202 :

ich merk', man soll kein Ding

zweimal erleben wollen.

Weidenstamm emploie pour désigner son trouble les termes Ekel, Scham, Qual, Verwirrung. Ce désarroi, qui peut déboucher sur le nihilisme, sera quelques années plus tard (1907) le thème des Briefe des Zurückgekehrten, Prosa II, S. Fischer Verlag, Berlin, 1973, pp. 321-357.

59. Dans la réalité : Philippe César Lanti, fils naturel de Casanova, né en 1744.

60. «Cesarino, den Abenteurer spiegelnd über den Abgrund der Zeit hinweg», Aufzeichnungen, op. cit., p. 219.

61. Op. cit., p. 249 :

Er macht Musik aus allem, was er anrührt!

p. 248 :

Wenn ich Musik gehört hab', ist mein Ohr

so voller Nachklang, dass ich Harmonien

der Spharen spüre [...].

62. Idem., p. 241.

63. «Cesarino (steht) kritisch und erfüllend zu seinem Spiegelbild», Aufzeichnungen, op. cit., p. 223.

64. Idem., p. 218 : «der schicksallos gewordene Vater und Autor».

65. Hofmannsthal, pour montrer le moment d'hésitation, puis la rapidité d'exécution note comme jeux de scène : verlegen, befangen... puis rasch et schnell, op. cit., pp. 253-257.

Idem., p. 257.

Vittoria : Wie Du sie verstehst,

die Kunst zn enden! Wer das kann, kann alles.

66. Op. cit., p. 259 : «Nur eine Sekunde lang überlegte Casanova, an wen ihn Lorenzi erinnerte. Dann wusste er, dass es sein eigenes Bild war, das ihm, um dreissig Jahre verjüngt, hier entgegentrat. Bin ich etwa in seiner Gestalt wiedergekehrt? fragte er sich. Da müsste ich doch vorher gestorben sein...».

67. Idem., p. 278. 
68. Ibid., p. 296 : «Wir sind aus gleichem Stoff gemacht, Lorenzi, sind Brüder im Geiste, und so dürfen sich unsere Seelen ohne falsche Scham, stolz und nackt, gegenüberstehen».

69. Bon nombre des analyses de Paul Requadt dans son article «Sprachverleugnung und Mantelsymbolik im Werke Hofmannsthals» pourraient être reprises pour l'œuvre de Schnitzler. Deutsche Vierteljahresschrift für Literatur, Wissenschaft und Geisfesgeschichte, 29, 1955, pp. 255-283.

70. Op. cit., p. 313 : «herrlich in seiner Nacktheit wie ein junger Gott».

71. Idem., p. 313 : «Eine Fabel ist Jugend und Alter, dachte er... Bin ich nicht ein Gott? Wir beide nicht Götter?»

72. Ibid., p. 318 : «Traum, in dem er manchmal in einer rätselhaften Weise nicht Casanova, sondern Lorenzi, nicht der Sieger, sondern der Gefallene, nicht der Entfliehende, sondern der Tote war, um dessen blassen Jünglingsleib einsamer Morgenwind spielte; und beide, er selbst und Lorenzi, waren nicht wirklicher als die Senatoren in den roten Purpurmanteln [...]».

73. Ibid., p. 319 : «ein wenig freundlicher Raum mit der Aussicht auf die fensterlose Mauer eines gegenüberliegenden Hauses».

74. Ibid., p. 317 : «eine wahrhaft tierische Gier»...; p. 320 : «eine beinahe unanständige Gier».

75. Ibid., p. 322 : «er verstand es vielmehr, auch hier den Harmlosen und Liebenswürdigen zu spielen».

76. Ibid., p. 323 : «Brückenstege, unter denen die schwärzlichen Kanäle den ewigen Wassern zuzogen ».

77. Voir supra, note 2. Die Schwestern oder Casanova in Spa, dans Arthur Schnitzler, Die Dramatischen Werke, S. Fischer Verlag, 1962, II, pp. 650-738.

p. 733:

Ist Wand'rung nicht der Seele ewiger Ruf ? [...]

Heimat und Fremde - Worte tauben Klangs

Für den, der nicht, nach Bürgerart, bedrückt

Von Vorurteil, verschüchtert vom Gesetz

Und feig verstrickt im Wirrsal des Gewissens,

Sich Ordnung lügt ins Chaos seiner Brust,

Der aufgetanen Sinns und freier Seele -

Gleich unsereinem aus dem Stegreif lebt.

\section{RÉSUMÉS}

Casanova rentre à Venise. Rendez-vous de l'Aventurier avec lui-même. Retour impossible pour le Casanova de quarante ans de Hofmannsthal, confronté au miroir du temps, au reflet de ses doubles. Par fidélité à sa nature, il renoncera à Venise, où son fils, double idéal, perpétuera le mythe. Retour parodique pour le Casanova de Schnitzler qui ne retrouve Venise qu'au prix d'une double mort symbolique: reniement de son essence d'aventurier et meurtre de son double, donc du mythe même de Casanova.

Casanova kehrt nach Venedig zurück. Begegnung des Abenteurers mit sich selbst. Unmögliche Heimkehr für den hofmannsthalschen Casanova von vierzig Jahren; er sieht sich dem Spiegel der Zeit, dem Bild seiner Doppelgänger entgegengestellt. Seiner Natur treu bleibend entsagt er Venedig, wo sein Sohn, als sein vollendetes Ebenbild, den Mythos weiterleben lässt. Parodie der Heimkehr für den schnitzlerschen Casanova, der seine Rückkehr nach Venedig mit einem 
doppelten - symbolischen - Tod bezahlt: er verleugnet seine Abenteurernatur, tötet seinen Doppelgänger und damit den Casanova-Mythos selbst.

\section{AUTEUR}

GENEVIĖVE ROUSSEL

Université de Lille III 